\title{
Gold Open Access
}

University of Oxford

\section{Source}

University of Oxford. Open Access Glossary.

Gold OA is where a paper is published immediately as OA in an online journal and is therefore stored in the publisher's system.

Key points to note:

- To cover the costs of gold OA, publishers normally charge a fee to the author or their institution, known as the Article Processing Charge (APC).

- The version of the paper released as gold OA is the version of record: i.e. the publisher's final (formatted) version.

- Gold does not mean 'gold standard' or that it is 'better' than Green. 